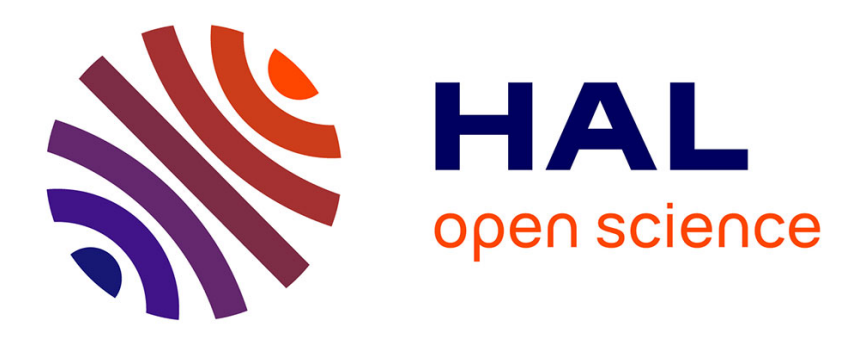

\title{
La vue est-elle souveraine dans l'esthétique paysagère?
}

Jean-François Augoyard

\section{To cite this version:}

Jean-François Augoyard. La vue est-elle souveraine dans l'esthétique paysagère?. Le Debat, 1991, 65,9 p. halshs-00380002

\section{HAL Id: halshs-00380002 \\ https://shs.hal.science/halshs-00380002}

Submitted on 29 Apr 2009

HAL is a multi-disciplinary open access archive for the deposit and dissemination of scientific research documents, whether they are published or not. The documents may come from teaching and research institutions in France or abroad, or from public or private research centers.
L'archive ouverte pluridisciplinaire HAL, est destinée au dépôt et à la diffusion de documents scientifiques de niveau recherche, publiés ou non, émanant des établissements d'enseignement et de recherche français ou étrangers, des laboratoires publics ou privés. 


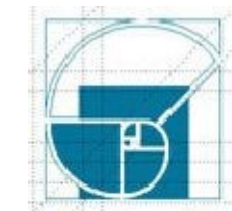

\section{cosessoll}

Jean-François Augoyard, philosophe et urbaniste, est notamment l'auteur de

Pas à pas. Essai sur le cheminement quotidien en milieu urbain, Paris, Le

Seuil,1979. Ses recherches actuelles portent en particulier sur

l'environnement sonore et sur l'esthétique de la lumière urbaine.

\section{La vue est-elle souveraine dans l'esthétique paysagère?}

Jean-François Augoyard.

- Article publié dans Le Débat. nº 65 mai-aout 1991.

- Chapitre XX, pp 334-345 in ROGER Alain (Ed) La théorie du paysage en France (1974-1994). Paris, Ed Champ, Vallon, 1995.

- Communication faite au Colloque international : Au-delà du paysage moderne, organisé par l'AREHESS, (Augustin Berque) à Paris, CCl, 19-20 octobre 1990. 
Pour citer ce document :

AUGOYARD, Jean-François. La vue est-elle souveraine dans l'esthétique paysagère ? Le Débat, mai-août 1991, n65, 9 p.

\section{- A voir...ou à entendre? -}

Le paysage n'est-il qu'à voir? Expérience naive : que chacun essaye de se remémorer un paysage connu ; plus encore, d'en évoquer le teneur sensitive. Parions que le sensatum sera essentiellement du visible : cette silhouette sinueuse et embrumée des montages sur fond d'azur, cette pénombre légèrement pailletée de lumière sous le saules qui bordent la rivière, cette chute de tuiles brunes qui, rebondissant de toits en toits, n'en finit pas de raconter l'immémoriale urbanité. Dans notre culture, la seule énonciation du mot "paysage" entraîne l'exercice du voir. A ce point que nombre d'ouvrages et d'articles traitant du paysage ne prennent même pas la peine de préciser que ce qui va être dit de l'environnement ou du milieu, sera proféré au nom de l'oeil, n'aura de vérité qu'au seul titre du regard. Remarque inaugurale : la souveraineté du voir dans la perception du paysage est tellement claire qu'elle va de soi, tellement habituelle qu'on tient pour évident que la logique du paysage visible - de sa topique devrait-on plutôt dire - , est aussi celle des autres sens. Voici deux questions inévitables La première : de quelle topique parlons nous? La deuxième : qu'est ce que les autres sens ont à voir avec le paysage?

De quelle topique parlons-nous? Les grands caractères du paysage moderne, fruit d'une élaboration achevée à la fin de la renaissance, sont aujourd'hui bien connus ${ }^{1}$. Je les rappelle en trois mots : distanciation, représentation spatialisante, artéfaction. Distanciation : l'oeil et le sujet regardant sont hors champ. Le point de vue suppose que le regardeur se retire du vu. Le paysage est le produit d'une objectivation du milieu, d'une opération de sélection, d'une mise en face de soi. Représentable, ensuite, par les outils de la spatialité géométrique, élaboré par la perspective "à l'italienne", le paysage se fonde sur un espace newtonien. L'artéfaction, enfin, ou artialisation (Roger 1978), c'est l'organisation du voir le paysage comme si c'était une oeuvre dont l'organisation est repérable. Le regardeur dont l'oeil est régi par une esthétique de la contemplation devient, au sens étymologique, spectateur. La notion de paysage occidental et moderne parait donc entièrement construite à partir d'une expérience du regard.

Seconde question : s'il est convenu que dans notre culture l'oeil prédomine, on ne sait quel est le rapport que ce sens entretient avec les autres, sinon à titre de vagues hypothèses. On ignore encore beaucoup de choses sur la perception auditive, olfactive, et même visuelle in situ, tant la psychophysiologie a privilégié jusqu'à présent l'étude expérimentale en laboratoire2 2 On sait encore moins s'il est possible de parler de paysage olfactif, de paysage tactile ou de paysage sonore par exemple.

L'idéal serait de traiter la question sens par sens De suivre exclusivement les procédures, les modes, les voies qui sont propres à chacun d'eux. A parcourir le même paysage avec un sens chaque fois différent, à fermer les yeux, puis se boucher les oreilles, etc... qu'adviendrait-il de mes convictions perceptives? Devrais-je reconsidérer l'espace, le temps, les catégories opératoires, mais aussi les catégories de l'analyse anthropologique?3

Or, une exploration de ce type sur l'écoute de l'environnement est en cours depuis quelques 
Pour citer ce document :

AUGOYARD, Jean-François. La vue est-elle souveraine dans l'esthétique paysagère ? Le Débat, mai-août 1991, n65, 9 p.

années. Il s'est passé en effet quelque chose d'étrange à propos du paysage. dans les années 70 : l'apparition du terme "paysage sonore". L'idée qu'un paysage pourrait être de l'audible. Pur paradoxe, pure métaphore? A voir... Ou plutôt, à entendre. Opposons donc le sens au sens. Suivons le jeu de près cette hypothèse. Filons les avatars de l'analogie proposée par les paysagistes sonores.

En présentant cette hypothèse du paysage sonore, j'adjoindrai comme matière à argumentation un ensemble de remarques issues de dix années d'études et recherches pluridisciplinaires sur l'environnement sonore4. Ma contribution à l'interrogation d'Augustin Berque sur un "au delà du paysage moderne" suivra comme guide deux questions

Le paysage sonore répète-t-il simplement le paysage visuel ou indique-t-il des éléments, des dimensions oubliées ou mésestimées dans le paysage moderne tel que vu?

La perception auditive de l'environnement, moins étudiée, moins investie de tradition dans notre culture peut-elle figurer parmi les paradigmes d'un paysage au-delà du moderne?

\section{- Soundscape -}

L'évolution de l'écoute au cours de siècle a deux causes essentielles : d'une part la diffusion de bruits plus intenses et surtout plus continus, d'autre part, le mélange des genres, la compénétration à la fois physique et sociale des milieux sonores. Pendant que l'environnement urbain se musicalise par l'envahissement des media sonores, l'invention et la pratique musicales deviennent volontiers bruitistes 5 . Musiciens, architectes, paysagistes, plasticiens, scénaristes et performers cultivant le design sonore, : ce sont des acteurs très divers qui, chacun de leur côté, vont contribuer au même résultat6. Il s'agit d'introduire la dimension esthétique dans la représentation contemporaine de l'environnement sonore. A cette entreprise il manquait un concept clé. Ce fut, dans les années 70 , celui de paysage sonore.

Un très important effort de définition et d'illustration de la notion fut proposé par Robert Murray-Schafer, l'inventeur du significatif néologisme : Soundscape. Dans ses différents ouvrages, mais aussi dans certaines de ses compositions, ainsi que dans les superbes programmes de paysages sonores qui sont les premières pierres d'un ambitieux "World Soundscape Project", le musicien canadien, volontiers méthodologue, ethnographe et mythologue, construit la représentation de l'environnement sonore comme on ferait d'une composition musicale $\mathrm{Ce}$ faisant, il donne à entendre la réalité comme une oeuvre de la nature. En ce sens, le paysage sonore désigne précisément ce qui dans l'environnement sonore est perceptible comme unité esthétique 7 .. Processus d'artialisation ; les formes ainsi perçues sont appréciables parce qu'elles ont l'air assujetties à une composition. Le meilleur accès à la compréhension du Soundscape est donc l'écoute de paysages sonores extraits de l'environnement, formatés, mis en scène à travers le support électroacoustique. Ainsi, autrefois, le tableau guida-t-il la propédeutique d'un nouveau regard sur le paysage visuel. 
Pour citer ce document :

AUGOYARD, Jean-François. La vue est-elle souveraine dans l'esthétique paysagère ? Le Débat, mai-août 1991, n65, 9 p.

\section{- Du temps qualifié -}

Quels sont les caractères remarquables du paysage sonore? La réponse à cette question commence nécessairement par l'expérience sensible. Ecoutons un des plus superbes paysages sonores que Murray-Schafer ait enregistré à Vancouver : Entrance in the Harbour. Au début, un clapotis à peine audible et, soudain, un grave et puissant appel de corne de brume qui emplit l'espace et le temps de l'écoute, qui transporte le corps. D'emblée le climat portuaire est là tout entier, vaste humide, d'une clarté laiteuse D'autres cornes et trompes, puis un passage d'avion, plus tard une cloche dessinant les contours plus nets de la terre qui avance, vont bientôt épeler l'espace, lui donner des repères, répondre au premier appel en tissant un polyphonie fort savante et étonnement musicale8

La perception de cette séquence sonore nous prend quatre minutes. Sans cette durée minimale, ni ma sensation ne réussirait pleinement à retrouver par évocation les voies de l'expérience des vastitudes brumeuses et humides, ni mon sentiment d'être dans un site, dans un lieu, ne serait accompli, ni enfin, mon jugement de goût - ici porté vers le sublime - n'aurait le temps de s'installer. En-deçà même de la reconnaissance objectale9 ${ }^{9}$ l'écoute d'un paysage sonore engage de manière fondamentale une accumulation de ce que je viens d'entendre. Ce caractère est propre à toute perception sonore. Par nature, le son est du temps qualifié. Pour toute écoute élémentaire et afortiori pour un ensemble complexe, c'est la rétroaction qui vient petit à petit construire le sens de ma perception. Au niveau élémentaire; celui de l'objet sonore simple, la rétroaction est rendue nécessaire par une limite physiologique : le seuil d'intégration temporelle. Au niveau complexe, la rétroaction est de nature connotative : ainsi, tant que la corne de brume n'a pas résonné plusieurs fois, ce bruit rose au rythme aléatoire que j'entends au début de "Entrance in the Harbour" n'est pas encore un clapotis de vagues ${ }^{10}$. Ce que j'entends du paysage ne serait donc pas fondamentalement une organisation spatiale mais une organisation temporelle. Il faut alors s'interroger sur la nature de l'espace sonore.

\section{- Discrétisation -}

L'espace sonore est un espace discret (au sens latin de discretum). Il n'implique fondamentalement ni la contiguitté, ni l'homogénéité. Le premier signe en est la discontigüité. On a pu ainsi soutenir que la perception sonore d'une rue se dessinerait comme un couloir seulement percé de fenêtres intermittentes ${ }^{11}$. La distribution des formes sonores d'un lieu ne correspond pas nécessairement à ce que l'organisation visuelle nous donne à voir. Un tel processus est conditionné par un gommage -l' oubli porte ici sur des sons- dont nous avons essayé de montrer l'extrême importance dans l'organisation des rhétoriques quotidiennes ${ }^{12}$. La carte sonore d'une ville telle que se la représente un habitant urbain, se dessinerait comme un ensemble d'isolats, d'éléments discrets, sans relation de hiérarchie topique, ni repérage selon les coordonnées cartésiennes. C'est un çà et là organisé selon une logique qualitative. On regroupe et on rapproche ainsi des lieux au titre de leur 
Pour citer ce document :

AUGOYARD, Jean-François. La vue est-elle souveraine dans l'esthétique paysagère ? Le Débat, mai-août 1991, n65, 9 p.

qualité sonore : aire des lieux bruyants, aire des lieux tranquilles.

Une autre manifestation de la discrétion de l'espace sonore est la disjonction toujours possible entre l'entendu et l'identifié. Dans le même moment, l'habitant perçoit et le son de cette cloche qu'il reconnaît venir de telle rue et le drône, le continuum sonore, qu'il peut n'avoir ni identifié, ni localisé clairement. Ces extrapolations, ces coalescences ne paraissent étonnantes que du point de vue de l'oeil. En fonction des sources et des configurations architecturales, les lois physiques de la propagation sonore vont commander, soit une audition de points discrets, disséminés, soit au contraire la superposition des sons étrangers les uns aux autres, soit encore, pour les marcheurs urbains, un réseau d'itinéraires sonores : chemin des oiseaux, chemins des métiers, chemins de l'eau. A être seulement entendu, le paysage perd donc les caractères intrinsèques de la spatialité newtonienne : continuité, homogénéité, isotropie. Comment cette structure paysagère peut-elle alors advenir? ? Peut-on observer les modalités par lesquelles un espace sonore se fait et se défait? Qu'arrive-t-il quand le son fait le lieu?

\section{- L'art du paysage sonore -}

Souvenons-nous du paysage évoqué tout à l'heure. Peut-on décrire cette perspective sonore? Où situer exactement le point de fuite? Qu'est ce qu'une ligne sonore? La transposition s'arrête court. Malgré les nombreuses tentatives qui jalonnent l'histoire de la culture humaine, y compris en musique, il faut reconnaître que toute métaphore graphique d'un phénomène sonore reste contingente et réductrice. Est-il plus fructueux d'utiliser des règles d'agencement, des catégories perceptives telles que figure et fond, par exemple?

Pour Murray-Schafer, la composition d'un paysage sonore fonctionne sur le même principe que le paysage visuel, à savoir le rapport entre figure et fond. Cette relation est éclairante dans l'intelligibilité d'un paysage de "haute-fidélité," c'est à dire audible dans sa musicalité naturelle comme un concert de carillon et de vols de pigeons dans une petite ville italienne un dimanche matin-, ou bien un paysage sonore enregistré et formaté dans une visée esthétique, comme c'est le cas pour l'entrée dans le port de Vancouver. Sous le néologisme "soundscape" demeure la fascination du landscape. Notons d'abord que ces catégories de figure et fond sont empruntées à le psychologie de la forme laquelle, comme on sait, a surtout analysé la perception visuelle. Par ailleurs et dans un but didactique louable, Murray-Schafer propose en effet de classer, dans l'ordre de l'excellence, d'une part les paysages sonores $h i$ - $f$, d'autre part, les paysages sonores low-fi, bruyants ou embrumés, si caractéristiques des villes. Ce tri reprend les critères cartésiens de la bonne représentation : clarté et distinction. C'est la chasse aux parasites, aux brouillages du signal et du sens. Qu'arrive-t-il quand la composition sonore est représentée par les catégories de l'intuitus? L'essence même du phénomène sonore est-elle encore rendue? En toute rigueur, un environnement sonore compris comme un paysage moderne ne devrait-il pas suivre les règles de la perception visuelle? 
Pour citer ce document :

AUGOYARD, Jean-François. La vue est-elle souveraine dans l'esthétique paysagère ? Le Débat, mai-août 1991, n65, 9 p.

\section{- Métaboles -}

Voici probablement le plus difficile problème posé par l'usage universalisant du terme de paysage sonore14. En fait, nous touchons à la mise en question de la pertinence du rapport figure/fond pour analyser un paysage sonore ordinaire. Dans l'urbaine quotidienneté, souvent encombrée par la compétition physique et sociale entre sources sonores multiples, le rapport entre fond et figure est beaucoup moins stable. Ce qui me paraissait figure entre plus tard dans le fond d'où émergent alors d'autres formes discernables. Ce phénomène est parfaitement décrit par une figure de la rhétorique classique. La métabole désigne ainsi un processus dans lequel les éléments d'un ensemble entrent en rapport de permutations et de combinaisons hiérarchisées sans qu'aucune configuration ne soit durable.

Par nature, l'espace sonore ne serait-il pas un espace métabolique? Les espaces sonores ordinaires supposent la métabole que ce soit par l'organisation des sources, ainsi le charmant fouillis sonore d'un marché, ou que ce soit par l'organisation perceptive elle-même, ainsi mon écoute, changeante au gré des jours, d'une même sonnerie, ainsi la possibilité de viser dans un même "bruit" intense des composantes différentes. Comme pour le premier caractère de discrétisation évoqué tout à l'heure, c'est encore le temps qui commande l'organisation complexe de l'environnement sonore. Les catégories perceptives de figure et fond ne sont-elles pas trop liées à une spatialité visuelle? Celles de durée et d'événement, ressortissant au régime du temps conviendraient sans doute mieux. Faut-il à ce prix abandonner la notion de paysage sonore ou en limiter l'usage aux (re)productions artistiques intentionnelles? Mais la question plus générale de la nature de l'espace sonore reste posée.

\section{- La pregnance du sonore -}

Nous savons d'expérience combien la musique mais aussi un paysage sonore in situ peuvent nous transporter. Etre absorbé, exorbité par le bruit de la mer, de l'orage, d'un foule en délire. Etre possédé par le timbre d'une voix ou son seul souvenir. Comment un son peut-il être objectivé? La distanciation spatiale si nécessaire à la construction du paysage visuel n'est ici d'aucun secours. Le retrait de l'auditeur, son absence du "cadre" ne construiront aucune organisation focale ni rien d'analogue à la veduta. L'auditeur ne réussit à s'abstraire d'un paysage sonore qu'en tombant dans l'ubiquité. Il devient une sorte d'oreille off. En fait, il ne peut avoir son lieu précis qu'à l'intérieur du représenté sonore 15 . In situ comme en écoute médiatisée, toute la question de la place du sujet de la perception sonore est de savoir comment s'organise pour lui le jeu du proche et du lointain dans le paysage. Léonard de Vinci, soupçonnant déjà les discrépances entre le visible et l'audible l'avait pressenti en ces termes : "Je me suis toujours demandé si un faible bruit rapproché peut paraître aussi fort qu'un grand bruit lointain."

Dans l'espace sonore, le jeu du proche et du lointain prend le pas sur la distinction entre le sujet et l'objet. Physiologiquement, de la naissance jusqu'à la mort, j'ouïs constamment le monde. En 
Pour citer ce document :

AUGOYARD, Jean-François. La vue est-elle souveraine dans l'esthétique paysagère ? Le Débat, mai-août 1991, n65, 9 p.

examinant l'identité singulière ou l'enveloppe du soi, on constate que la limite est très poreuse. Comme le montre l'analyse de l'expérience enfantine de l'écho, l'"intervalle sonore du soi"(Lecourt, 1986) 16 est en effet constitué à partir d' une série de distinctions audibles qui opposent de manière dynamique l'intérieur à l'extérieur, le subjectif à l' objectif, le proche au lointain, chacun des termes ayant d'autant plus besoin de l'autre pour définir sa propre existence qu'il est incarné dans une matière sonore variable et changeante par nature.

\section{- L'écoute immanente -}

Ainsi est construite la bipolarité : d'une part, vulnérabilité à la pregnance sonore, de l'autre, capacité étonnante d'expression du soi. Si le monde et l'autre peuvent facilement pénétrer phoniquement dans ma privauté, voire dans mon corps propre - et les voix de la paranoïa étudiées en psychopathologie disent cette vulnérabilité de l'écoute -, en contrepartie je suis doué d'un pouvoir sonore potentiellement équivalent. Actions expressives sur l'environnement : faire peur par un seul cri, imprimer aux lieux et aux jours mes marques sonores. Ou actions de synthèse perceptive : opérer des sélections sémantiques dans un ensemble sonore confus, réussir à ne pas "entendre" les bruits les plus éclatants. Facilement oublié quand il ne me touche pas, volontiers envahissant quand il m'affecte, l'environnement sonore est très lié à l'ordre de l'expressif et à l'ordre du pathos.

En définitive, le paysage entendu diffère du paysage vu à trois titres : il est une topique de la discrétion; il est une composition métabolique, il est enfin une représentation jamais déliée du pathique, jamais libérée de l'immanence du sujet. Ces qualités sans lesquelles l'espace sonore n'advient pas, on voit aisément qu'elles ne doivent pas grand chose à la rationalité moderne forgée à la renaissance, more geometrico, et qui présida à l'organisation du regard paysager. Faut-il en déduire une radicale différence entre la logique du voir et celle de l'entendre? Ou plus encore, que l'espace synesthésique ne serait qu'une fiction, qu'il n'existe qu'une concurrence de diverses perceptions de l'environnement, sans lien entre elles? La confrontation du paysage sonore et du paysage visuel esquissée ici devrait être poursuivie en deux façons. Parce qu'elle suscite la question du rééquilibrage des perceptions de l'environnement. Parce qu'elle peut mettre en cause le statut du paysage moderne. Je voudrais simplement en indiquer les pistes en guise de conclusion.

\section{- Vers un rééquilibrage des sens? -}

L'exemple remarquable du paysage sonore laisse-t-il présager un ébranlement de la citadelle du visible.? Assiste-t-on à un rééquilibrage des sens dans la perception de l'environnement? En me gardant de toute vaticination hasardeuse, j'évoquerai seulement quelques indices.

A noter d'abord que l'apparition de l'idée de paysage sonore est à prendre comme l'émergence savante d'un intérêt croissant de l'opinion commune pour l'environnement sonore. Cette préoccupation induite essentiellement par les nuisances du bruit n'apparaît pour l'instant que dans 
Pour citer ce document :

AUGOYARD, Jean-François. La vue est-elle souveraine dans l'esthétique paysagère ? Le Débat, mai-août 1991, n65, 9 p.

les pays industrialisés. Mais le déplacement des objets quérulents serait à interroger en lui-même. Pourquoi les odeurs et les servitudes de passage au XIXéme, pourquoi le bruit aujourd'hui? Soucieuse de remettre dans une perspective plus large les nombreux travaux de psycho-acoustique centrés depuis des années sur la nuisance, ou sur l'écoute musicale, une anthropologie culturelle des phénomènes sonores est donc en voie de constitution. Elle inclut la sociologie de l'environnement et une esthétique sonore générale17

Il existe d'autres signes indiquant la possibilité d'un rééquilibrage culturel des sens C'est d'abord l'actuel regain d'intérêt scientifique pour la fonction cognitive des sens 18 et pour la question des corrélations sensorielles. Touchant ce dernier thème, l'éclipse a été longue puisque rien n'était venu remettre sérieusement en cause la fameuse et problématique théorie des correspondances qui avait passionné le XIXéme siècle. Citons encore le thème du retour ou de la redécouverte des sens plus archaïques tenus en sommeil par la domination du visible. L'audition et le tact retrouveraient ainsi une valeur remarquable due précisément à leur précédence temporelle. Ce statut paradigmatique par rapport aux autres sens, dont l'oeil, est analysé soit du point de vue ontogénétique (stade in utero : Tomatis, Lasserre) ${ }^{19}$, soit dans l'histoire naturelle et la géographie des anciens écosystèmes pré-urbains et visuellement opaques (milieux sylvestres), soit à travers l' évolution sociale et culturelle des canaux de communication (Mac Luhan 20).

\section{- Un paysage à revoir -}

La question du rééquilibrage des sens passe sans doute par celle de la remise en cause du statut du paysage moderne. On peut faire l'hypothèse que les qualités générales de discrétion, de métabolisation et de prégnance qui nous paraissaient caractériser la perception auditive de l'espace ne sont pas propres à l'environnement sonore A côté d'un regard cultivé qui paysage l'environnement visible de façon "moderne", il existe un regard banal, insouciant des symétries, indemne d'une esthétique de la contemplation, heureusement incapable de recul, bref, une quotidienne manière d'être indissolublement dans le paysage et dont il est possible d'analyser la nature et l'efficace21. Cette attitude est "parasitologique" parce qu'elle s'oppose à la vision paysagère moderne et qu'elle en sape les fondements : plus de perspective géométrique, projection de soi dans le paysage, déréalisation des formes données, réhabilitation de l'action face à l'expectation. L'existence de cette vision banale passe inaperçue parce que ses instruments favoris appartiennent à une rhétorique des pratiques et non à l'ordre du discours. Or, par-delà les différences inhérentes à la nature physique des signaux et à la complexion propre à chaque organe, cette manière de voir suit une logique comparable à celle des manières d'entendre analysées plus haut.

Vision paysagère et audition paysagère entreront sinon en correspondance, $\mathrm{du}$ moins en équivalence fonctionnelle à partir du moment où seront réhabilités les critères de relation avec l'environnement sensible que la conception moderne du paysage masquait. C'est à partir de ces 
impératifs retrouvés que se dessinerait l'au-delà du paysage moderne. Premier impératif : une relation dialectique entre la dimension physique et la dimension phénoménologique de l'environnement perceptible. L'interdisciplinarité large et les concepts transversaux deviennent alors fort nécessaires dans l'ordre de l'explication. Deuxième impératif : la réinsertion du sujet dans le paysage. L'esthétique de la contemplation et du jugement de goût nous avait fait oublier à quel prix on s'absente du paysage. Troisième impératif : l'invention du paysage. L'esthétique de la mimésis nous a fait croire que le paysage était un donné naturel mis en scène par l'art, une perception close sans devenir. Pourtant, nous le savons de plus en plus, la perception de l'environnement fait partie de l'invention du quotidien 22.

Ce n'est pas la visibilité du paysage qui est à réformer, c'est une vision telle que dans le paysage moderne. Redécouvrir la pluralité des sens, rentrer dans le décor, réinventer le trop connu, voilà trois opérations indispensables pour réussir à revoir le paysage. Et, à nouveau, penser l'immanence. 